\title{
Preoperative physiotherapy and short-term functional outcomes of primary total knee arthroplasty
}

\author{
Mohd Shukry Mat Eil @ Ismail ${ }^{1,2}$, MD, MMed, Mohd Ariff Sharifudin ${ }^{2,3}$, MBBS, MMed, Amran Ahmed Shokri² ${ }^{2}$ MD, MMed,
} Shaifuzain Ab Rahman ${ }^{2}$, MD, MMed

INTRODUCTION Physiotherapy is an important part of rehabilitation following arthroplasty, but the impact of preoperative physiotherapy on functional outcomes is still being studied. This randomised controlled trial evaluated the effect of preoperative physiotherapy on the short-term functional outcomes of primary total knee arthroplasty (TKA).

METHODS 50 patients with primary knee osteoarthritis who underwent unilateral primary TKA were randomised into two groups: the physiotherapy group $(n=24)$, whose patients performed physical exercises for six weeks immediately prior to surgery, and the nonphysiotherapy group $(n=26)$. All patients went through a similar physiotherapy regime in the postoperative rehabilitation period. Functional outcome assessment using the algofunctional Knee Injury and Osteoarthritis Outcome Score (KOOS) scale and range of motion (ROM) evaluation was performed preoperatively, and postoperatively at six weeks and three months.

RESULTS Both groups showed a significant difference in all algofunctional KOOS subscales $(p<0.001)$. The mean score difference at six weeks and three months was not significant in the sports and recreational activities subscale for both groups $(p>0.05)$. Significant differences were observed in the time-versus-treatment analysis between groups for the symptoms $(p=0.003)$ and activities of daily living $(p=0.025)$ subscales. No significant difference in ROM was found when comparing preoperative measurements and those at three months following surgery, as well as in time-versustreatment analysis $(p=0.928)$.

CONCLUSION Six-week preoperative physiotherapy showed no significant impact on short-term functional outcomes (KOOS subscales) and ROM of the knee following primary TKA.

Keywords: algofunctional Knee Injury and Osteoarthritis Outcome Score, functional outcome, prehabilitation, preoperative physiotherapy, total knee arthroplasty

\section{INTRODUCTION}

Physiotherapy has long become the pivotal component of rehabilitation following total joint arthroplasty (TKA) and other orthopaedic procedures. However, limited information is available on the potential role of physiotherapy in preparation for surgery, as previous studies had diverse results. ${ }^{(1-8)}$ Prehabilitation, or the concept of preparing the body prior to a stressful event such as surgery, aims to develop patients' ability to withstand stress in order to maximise postoperative outcomes. Patients who undergo successful prehabilitation are expected to have shorter recovery times, less dependence on caregivers and faster recovery of preoperative functions. ${ }^{(2)}$

These positive results may have particular relevance to arthroplasty surgery, as the postoperative status of hip and knee arthroplasty patients is strongly related to preoperative functional status. ${ }^{(1)}$ In a review, preoperative physiotherapy was suggested to have some postoperative benefits for total knee or hip arthroplasty patients; however, the results were inconclusive due to lack of strong research evidence. ${ }^{(3)}$ The findings of another study had uncertain clinical significance and failed to support the routine use of preoperative physical therapy in knee replacement therapy. ${ }^{(4)}$ Results from other studies were inconclusive due to their small sample size and methodological limitations such as the use of less effective exercise intervention. ${ }^{(5-8)}$ This study was designed to evaluate the effect of preoperative physiotherapy on the short-term functional outcomes of primary TKA at three intervals - preoperatively, and postoperatively at six weeks and three months - in the course of treatment.

\section{METHODS}

A randomised controlled trial was conducted among patients with primary knee osteoarthritis at the Arthroplasty Clinic of Hospital Universiti Sains Malaysia (HUSM) in Kelantan, Malaysia, from 1 June 2010 to 10 August 2011. Institutional ethical approval was obtained before conducting the study. Patients were eligible to participate if they were (a) above the age of 45 years; (b) lived within a convenient distance of the physiotherapy facility; (c) had been diagnosed with unilateral or bilateral primary knee osteoarthritis; and (d) underwent unilateral TKA at HUSM. Patients with the following conditions were excluded: systemic inflammatory arthritis; degenerative joint diseases involving the hip or ankle joint or spine; medical comorbidities with an inability to tolerate a moderate level of physical exertion; premorbid knee joint stiffness; history of cardiovascular accident; and cognitive, psychological or language impairment. Sample size estimation was performed using PS version 3.0.34 (Dupont and Plummer, Nashville, TN, USA), based on a standard deviation of 11.4 and detectable

\footnotetext{
${ }^{1}$ Department of Orthopedics, Hospital Raja Perempuan Zainab II, ${ }^{2}$ Department of Orthopedics, School of Medical Sciences, Universiti Sains Malaysia, Kelantan, ${ }^{3}$ Department of Orthopaedics, Traumatology and Rehabilitation, Kulliyyah (Faculty) of Medicine, International Islamic University Malaysia, Pahang, Malaysia

Correspondence: Dr Mohd Shukry Mat Eil @ Ismail, Orthopaedic Surgeon, Department of Orthopaedics, Hospital Raja Perempuan Zainab II, 15586 Kota Bharu, Kelantan, Malaysia.mshukry76@gmail.com
} 
difference in the population mean of 9.1. The minimum computed sample size was 26 , with an estimated dropout rate of $10 \%$. Patient assignment into two groups was randomised according to the week of their surgery; those who were scheduled for surgery on odd and even weeks of the month were assigned to the physiotherapy and nonphysiotherapy groups, respectively. Patient assignment was performed by the primary investigator and this was not made known to the surgeons.

The use of the algofunctional Knee Injury and Osteoarthritis Outcome Score (KOOS) scale required no licence; the questionnaire was downloaded from the website http://www. koos.nu. ${ }^{(9)}$ The Malay version of the KOOS was translated from

Table I. Preoperative physiotherapy regime of the physiotherapy group.

\begin{tabular}{ll}
\hline Exercise & Description \\
\hline $\begin{array}{l}\text { Thermotherapy } \\
\text { Stationary bike }\end{array}$ & Hot pack for $10 \mathrm{~min}$ \\
$\begin{array}{l}\text { Ankle circulatory } \\
\text { exercise (ankle pumps) }\end{array}$ & Low-resistance cycling for $15 \mathrm{~min}$ \\
Isometric quadriceps & Hold for $5 \mathrm{~s}, 50$ repetitions \\
Inner range quadriceps & Hold for $5 \mathrm{~s}, 50$ repetitions \\
Hamstring stretch & Hold for $10 \mathrm{~s}$, gradually increase to \\
& $15 \mathrm{~s}$ as tolerated, 10 repetitions \\
Straight leg raise & Leg raised to $45^{\circ}, 20$ repetitions \\
Heel slides & Hold for $5 \mathrm{~s}, 20$ repetitions \\
\hline
\end{tabular}

the English version LK1.0 and validated according to Mapi Research Institute procedures for linguistic validation. ${ }^{(10)}$ It measured functional outcomes on the following five subscales: symptoms (seven items), pain (nine items), activities of daily living (ADL) function (17 items), sports and recreational function (five items) and quality of life (QOL) (four items). Sociodemographic and other medical data collected included associated medical comorbidities, affected area (unilateral or bilateral), duration of knee pain, and frequency of TKA and physiotherapy status. In the present study, KOOS subscales were used to assess short-term functional outcomes.

Preoperative assessment was done on both groups using the algofunctional KOOS and an evaluation of the range of motion (ROM) of the knee. The physiotherapy group performed physical exercises twice weekly over a six-week period immediately prior to surgery. Their physiotherapy regime included stretching, isometric strengthening exercises, mobilising exercises and heat therapy (Table I). The exercises performed were similar to those in the postoperative exercise protocol for TKA patients at HUSM (Table II). The knee arthroplasty surgery was performed by two senior orthopaedic surgeons using either the ADVANCE $\mathbb{R}$ Medial-Pivot Knee or NexGen ${ }^{\circledR}$ Complete Knee Solution Legacy ${ }^{\circledR}$ Knee Posterior Stabilized (LPS) LPS-Flex Fixed Bearing Knee implants. After surgery, all patients were given mechanical and pharmacological prophylaxis for deep vein thrombosis and underwent the same postoperative rehabilitation protocol.

Table II. Postoperative physiotherapy protocol of total knee arthroplasty patients at Hospital Universiti Sains Malaysia.

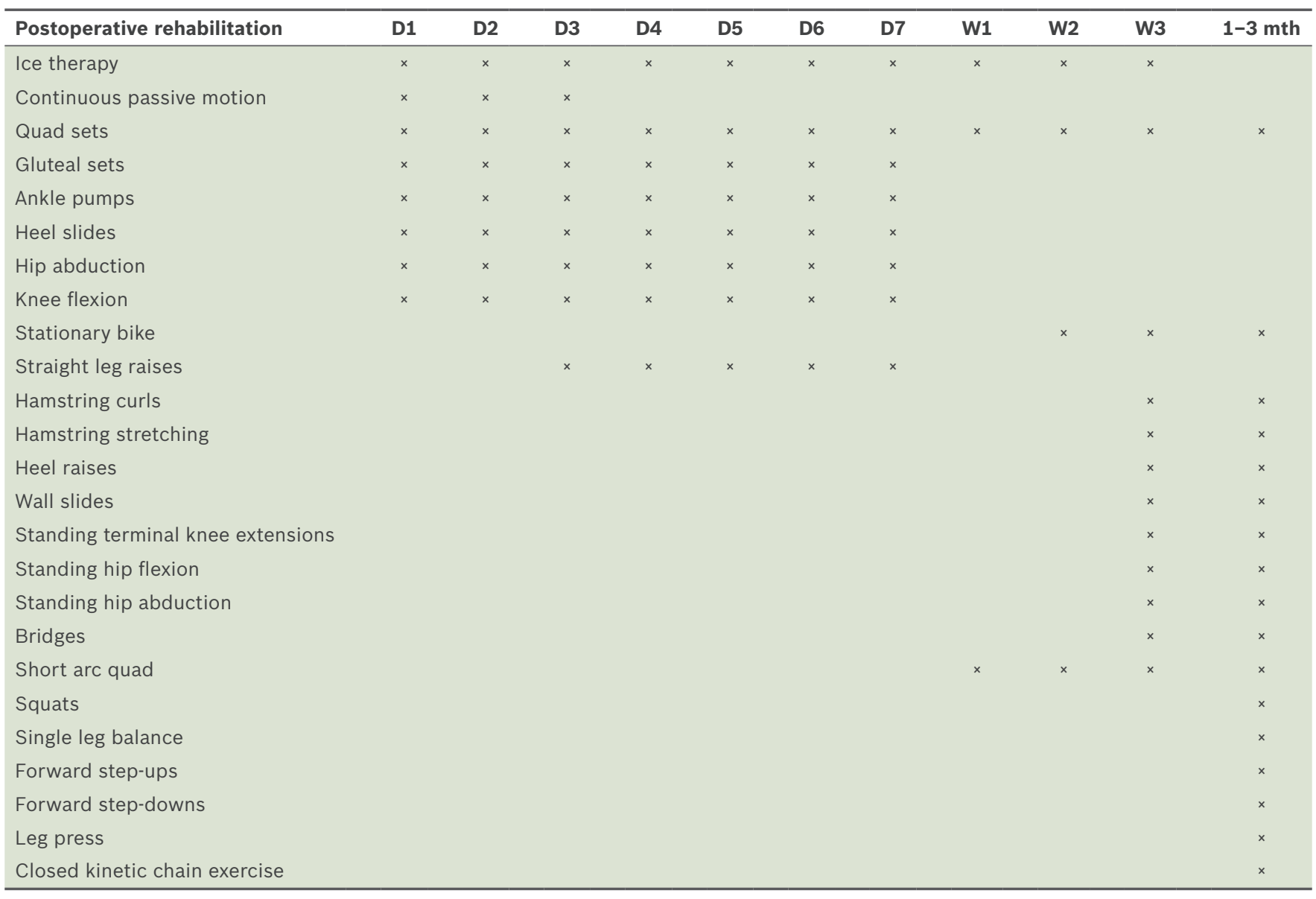

D: day; W: week 
Table III. Sociodemographic and medical data of the study population.

\begin{tabular}{|c|c|c|c|}
\hline \multirow[t]{2}{*}{ Characteristic } & \multicolumn{3}{|c|}{ No. (\%) } \\
\hline & Total $(n=50)$ & $\begin{array}{l}\text { Physiotherapy } \\
\text { group }(n=24)\end{array}$ & $\begin{array}{c}\text { Nonphysiotherapy } \\
\text { group }(n=26)\end{array}$ \\
\hline \multicolumn{4}{|l|}{ Gender } \\
\hline Male & $7(14)$ & $2(8.3)$ & $5(19.2)$ \\
\hline Female & $43(86)$ & $22(91.7)$ & $21(80.8)$ \\
\hline \multicolumn{4}{|l|}{ Affected knee } \\
\hline Bilateral & $48(96)$ & $24(100.0)$ & $24(92.3)$ \\
\hline Unilateral & $2(4)$ & 0 & $2(7.7)$ \\
\hline \multicolumn{4}{|l|}{ Duration of knee pain (yr) } \\
\hline$\leq 5$ & $31(62)$ & $15(62.5)$ & $16(61.5)$ \\
\hline$>5$ & $19(38)$ & $9(37.5)$ & $10(38.5)$ \\
\hline \multicolumn{4}{|l|}{ History of TKA } \\
\hline First time & $34(68)$ & $15(62.5)$ & $19(73.1)$ \\
\hline On contralateral knee & $16(32)$ & $9(37.5)$ & $7(26.9)$ \\
\hline \multicolumn{4}{|l|}{ Knee implant } \\
\hline ADVANCE & $44(88)$ & $21(87.5)$ & $23(88.5)$ \\
\hline NexGen & $6(12)$ & $3(12.5)$ & $3(11.5)$ \\
\hline \multicolumn{4}{|l|}{ Duration of TKA (hr) } \\
\hline$<2$ & $14(28)$ & $9(37.5)$ & $5(19.2)$ \\
\hline $2-3$ & $35(70)$ & $15(62.5)$ & $20(76.9)$ \\
\hline$>3$ & $1(2)$ & 0 & $1(3.8)$ \\
\hline Postoperative complication & $8(16)$ & $2(8.3)$ & $6(23.1)$ \\
\hline Surgical site infection & $4(8)$ & $2(8.3)$ & $2(7.7)$ \\
\hline Periprosthetic fracture & $1(2)$ & 0 & $1(3.8)$ \\
\hline Flexion contracture & $1(2)$ & 0 & $1(3.8)$ \\
\hline Anaemia-induced NSTEMI & $1(2)$ & 0 & $1(3.8)$ \\
\hline
\end{tabular}

*Age presented as mean. ADVANCE: ADVANCE ${ }^{\circledR}$ Medial-Pivot Knee; NexGen: NexGen ${ }^{\circledR}$ Complete Knee Solution Legacy ${ }^{\circledR}$ Knee Posterior Stabilized (LPS) LPS-Flex Fixed Bearing Knee; NSTEMI: non-ST elevation myocardial infarction; TKA: total knee arthroplasty

Postoperative evaluation was done at six weeks and three months following surgery using the KOOS, and the ROM of the operated knee was measured using a goniometer.

The primary investigator performed all data collection. Data was analysed using PASW Statistics for Windows version 18.0 (SPSS Inc, Chicago, IL, USA). Numerical variables were presented as mean \pm standard deviation, while categorical data was expressed in frequency and percentages. Repeated measures analysis of variance (ANOVA) was used to compare the pre- and postintervention values of all five subscales of KOOS in both groups.

\section{RESULTS}

Among the 50 patients recruited for the study, 86\% $(n=43)$ were female. The mean age of the study population was 64.6 (range 47.0-79.0) years and the age group distribution was equal in both treatment groups. One patient in the physiotherapy group had primary knee osteoarthritis and underwent surgery at the age of 47 years for severe pain. All patients who were recruited returned for follow-up. Patients in the physiotherapy group fully complied with the physiotherapy programme. The sociodemographic and medical data of the participants is summarised in Table III.

Repeated measures ANOVA performed within groups showed that there was a significant difference in mean score within all KOOS subscales when scores at different times were compared $(p<0.001)$. However, apart from the ROM score, there was no significant difference between postoperative scores at six weeks and those at three months in the nonphysiotherapy group (Table IV). Repeated measures ANOVA between group analyses with regard to time indicated that there was a significant difference in symptoms $(p=0.003)$ and ADL function $(p=0.025)$, but no significant difference was found for other KOOS subscales $(p=0.303$ for pain; $p=0.233$ for sports and recreational function; $\mathrm{p}=0.362$ for $\mathrm{QOL}$ ) and $\mathrm{ROM}$ measurements ( $p=0.928)$.

In the symptoms subscale, the mean score of the physiotherapy group did not overlap with the confidence interval $(\mathrm{Cl})$ of the nonphysiotherapy group at three months postoperatively, indicating that there was a significant difference in mean scores between the groups at that point. Similarly, the mean score of the physiotherapy group did not overlap with the $\mathrm{Cl}$ of the 
Table IV. Comparison of Knee Injury and Osteoarthritis Outcome Score subscales and knee range of motion (ROM) within treatment groups over time $(n=50)$.

\begin{tabular}{|c|c|c|c|c|c|c|}
\hline \multirow[t]{2}{*}{ Time } & \multicolumn{3}{|c|}{ Physiotherapy } & \multicolumn{3}{|c|}{ Nonphysiotherapy } \\
\hline & MD & $95 \% \mathrm{Cl}^{*}$ & p-value & MD & $95 \% \mathrm{Cl}^{*}$ & p-value \\
\hline \multicolumn{7}{|l|}{ Preoperation vs. 6 wk } \\
\hline Pain & -28.71 & $-38.20,-19.22$ & $<0.001$ & -23.21 & $-32.15,-14.27$ & $<0.001$ \\
\hline $\mathrm{ADL}$ & -21.33 & $-30.12,-12.55$ & $<0.001$ & -11.67 & $-19.48,-3.86$ & 0.002 \\
\hline QOL & -26.83 & $-34.69,-18.97$ & $<0.001$ & -20.40 & $-30.99,-10.94$ & $<0.001$ \\
\hline ROM & 17.92 & $8.68,26.98$ & $<0.001$ & 18.46 & $4.47,32.46$ & 0.007 \\
\hline \multicolumn{7}{|l|}{ Preoperation vs. $3 \mathrm{mth}$} \\
\hline Symptoms & -20.93 & $-29.42,-12.43$ & $<0.001$ & -8.81 & $-14.07,-3.54$ & 0.001 \\
\hline Pain & -36.13 & $-45.16,-27.10$ & $<0.001$ & -28.31 & $-37.41,-19.22$ & $<0.001$ \\
\hline ADL & -29.26 & $-37.23,-21.30$ & $<0.001$ & -17.71 & $-27.02,-8.40$ & $<0.001$ \\
\hline QOL & -35.43 & $-46.57,-24.28$ & $<0.001$ & -26.78 & $-37.40,-16.16$ & $<0.001$ \\
\hline ROM & 3.75 & $-4.15,11.65$ & 0.698 & 5.39 & $-4.60,15.37$ & 0.536 \\
\hline \multicolumn{7}{|l|}{6 wk vs. 3 mth } \\
\hline Symptoms & -8.41 & $-14.52,-2.30$ & 0.005 & -1.37 & $-5.36,2.62$ & $>0.950$ \\
\hline Pain & -7.42 & $-13.59,-1.25$ & 0.015 & -5.10 & $-10.99,0.786$ & 0.106 \\
\hline ADL & -7.93 & $-14.34,-1.52$ & 0.012 & -6.04 & $-13.98,1.91$ & 0.188 \\
\hline Sports and recreation & -3.13 & $-8.44,2.19$ & 0.428 & -2.69 & $-6.98,1.59$ & 0.358 \\
\hline QOL & -8.60 & $-15.92,-1.27$ & 0.018 & -5.81 & $-13.47,1.85$ & 0.189 \\
\hline ROM & -14.17 & $-21.44,-6.89$ & $<0.001$ & -13.08 & $-22.39,-3.77$ & 0.004 \\
\hline
\end{tabular}

*Data presented as lower and upper limit. Repeated measures analysis of variance was applied within groups, followed by a pairwise comparison, with confidence interval $(\mathrm{Cl})$ adjustment using the Bonferroni correction. ADL: activities of daily living; QOL: quality of life; MD: mean difference

nonphysiotherapy group at six weeks and three months in the ADL function subscale. However, in the QOL subscale, the mean score of the physiotherapy group overlapped with the $\mathrm{Cl}$ of the nonphysiotherapy group preoperatively, and postoperatively at six weeks and three months, indicating no significant difference. Even though the mean scores of each group in the sports and recreational subscales did not overlap with the Cls of the other group (Table $\mathrm{V}$ ), the differences were not significant between the groups $(p=0.233)$.

Repeated measures ANOVA performed within groups showed that mean ROM scores were significantly different when scores at different times were compared $(p<0.001)$; when performed between the groups, mean ROM scores were not significantly different $(p=0.928$ ). Furthermore, there was no significant difference between preoperation and three-month ROM in both groups (Table IV). The mean score of the physiotherapy group and $\mathrm{Cl}$ of the nonphysiotherapy group overlapped at preoperation and six weeks, which indicated no significant difference between the groups (Table V).

\section{DISCUSSION}

The majority of the patients were female. This was consistent with the criterion of osteoarthritis, which has a strong association with the female gender and advanced age. ${ }^{(11)}$ More than half of the patients experienced knee pain for less than five years; however, the duration of symptoms does not always correlate with the severity of osteoarthritis. A total of 40 patients had associated comorbid illness at the time of surgery, correlating with previous evidence showing that comorbidities could increase the risk of infection. ${ }^{(12)}$ Preoperative physiotherapy did not shorten the surgical time of TKA. The majority of patients from both groups underwent surgery within 2-3 hours $(62.5 \%$ in the physiotherapy group, and $76.9 \%$ in the nonphysiotherapy group). The postoperative complication rate was higher in the nonphysiotherapy group (23.1\%) compared to the physiotherapy group $(8.3 \%)$, and two patients from each group had superficial surgical site infection (SSI). At the time of surgery, 40 patients had associated comorbid illnesses; this correlates with previous evidence that comorbidities could increase the risk of infection. ${ }^{(12)}$ In this study, all of the patients who developed SSI and postoperative wound dehiscence had underlying diabetes mellitus at the time of surgery. Otherwise, the incidence of postoperative complications in this study was comparable with that of other studies. ${ }^{(13,14)}$

Overall analysis of the five subscales of the KOOS showed that significant improvement was seen after TKA in both groups. Patients who underwent TKA had significant improvement in symptom and functional outcomes, regardless of whether they underwent preoperative physiotherapy or not. The statistical results for KOOS subscales correlated with previous reports suggesting that TKA improved functional status, relieved pain and resulted in relatively low perioperative morbidity. ${ }^{(13)}$ Findings for the sports and recreational subscale were consistent with the results of another report on local osteoarthritis populations. ${ }^{(15)}$ 
Table V. Comparison of mean Knee Injury and Osteoarthritis Outcome Score subscales and knee range of motion (ROM) between the treatment groups over time $(n=50)$.

\begin{tabular}{|c|c|c|c|c|}
\hline \multirow[t]{2}{*}{ Time } & \multicolumn{2}{|c|}{ Physiotherapy } & \multicolumn{2}{|c|}{ Nonphysiotherapy } \\
\hline & Mean & $95 \% \mathrm{Cl}^{*}$ & Mean & $95 \% \mathrm{Cl}^{*}$ \\
\hline \multicolumn{5}{|l|}{ Preoperation } \\
\hline Symptoms & 75.71 & $69.78,81.62$ & 81.40 & $75.71,87.09$ \\
\hline Pain & 58.23 & $51.44,66.26$ & 59.74 & $53.22,66.26$ \\
\hline ADL & 60.24 & $53.97,66.52$ & 62.85 & $56.82,68.88$ \\
\hline $\begin{array}{l}\text { Sports and } \\
\text { recreation }\end{array}$ & 23.96 & $19.26,28.66$ & 24.62 & $20.10,29.13$ \\
\hline QOL & 28.66 & $23.98,33.34$ & 30.79 & $26.29,35.29$ \\
\hline ROM & 112.50 & $105.32,119.69$ & 107.69 & $100.79,114.60$ \\
\hline \multicolumn{5}{|l|}{ Postoperation } \\
\hline \multicolumn{5}{|l|}{$6 w k$} \\
\hline Symptoms & 88.22 & $82.99,93.45$ & 88.84 & $82.99,93.45$ \\
\hline Pain & 86.94 & $81.06,92.82$ & 82.95 & $77.30,88.60$ \\
\hline$A D L$ & 81.58 & $75.37,87.78$ & 74.52 & $68.56,80.48$ \\
\hline $\begin{array}{l}\text { Sports and } \\
\text { recreation }\end{array}$ & 39.58 & $34.35,44.82$ & 32.12 & $27.09,37.14$ \\
\hline QOL & 55.49 & $49.93,61.05$ & 51.76 & $46.42,57.10$ \\
\hline$R O M$ & 94.58 & $88.05,101.12$ & 89.23 & $82.95,95.51$ \\
\hline \multicolumn{5}{|l|}{$3 \mathrm{mth}$} \\
\hline Symptoms & 96.63 & $92.36,100.90$ & 90.21 & $86.10,94.31$ \\
\hline Pain & 94.35 & $90.49,98.23$ & 88.06 & $84.34,91.77$ \\
\hline$A D L$ & 89.50 & $85.04,93.97$ & 80.56 & $76.27,84.84$ \\
\hline $\begin{array}{l}\text { Sports and } \\
\text { recreation }\end{array}$ & 42.71 & $37.14,48.27$ & 34.81 & $29.46,40.15$ \\
\hline QOL & 64.08 & $57.08,71.08$ & 57.57 & $50.84,64.29$ \\
\hline ROM & 108.75 & $104.68,112.82$ & 102.31 & $98.40,106.22$ \\
\hline
\end{tabular}

* Data presented as lower and upper limit. Multivariate test was performed for time $\times$ treatment effect, Pillai's Trace $<0.05$. Repeated measures analysis of variance was applied within groups with regard to time, followed by a pairwise comparison, with confidence interval $(\mathrm{Cl})$ adjustment using the Bonferroni correction. Assumption of normality, homogeneity of variances and compound symmetry were checked and fulfilled. ADL: activities of daily living; QOL: quality of life

Statistical results for ROM showed that preoperative physiotherapy did not have a significant impact on postoperative knee ROM, as both groups showed no significant differences between ROM measurements preoperatively and at three months (Table IV). A single factor such as preoperative physiotherapy alone was not able to influence postoperative knee flexion. Generally, knees with good preoperative flexion have better flexion postoperatively than those with poor preoperative flexion. ${ }^{(16)}$ Other factors that affect postoperative knee flexion in TKA include implant design, preoperative ROM, the surgical technique used, knee kinematics, associated perioperative complications and compliance to postoperative rehabilitation. ${ }^{(17)}$ The findings of this study suggested that a combination of strengthening and mobilising preoperative physiotherapy did not improve postoperative shortterm functional outcomes of primary TKA. Patients who underwent TKA experienced significant improvements in functional outcomes and knee ROM at various intervals, whether or not they were involved in preoperative physiotherapy (Table IV).

Our study had a few limitations, including its small sample size due to logistical reasons and interventions that might not be suitable for elderly patients accustomed to a sedentary lifestyle.

Previous studies reported similar insignificant results. In a randomised trial involving 39 patients by Weidenhelm et al, the preoperative physiotherapy regime used did not demonstrate any major benefits in patients $(n=19),{ }^{(5)}$ while D'Lima et al suggested that a short duration of physical therapy is not sufficient for patients with chronic arthritis. ${ }^{(6)}$ A trial involving 108 patients (including 45 patients for TKA) revealed that both the physical therapy and control groups for TKA showed a similar response. ${ }^{(7)}$ A systematic review of five randomised controlled trials concluded that preoperative physiotherapy programmes were not effective in improving outcomes after TKA, although their effect on total hip replacement cannot be adequately determined. ${ }^{(8)}$

In conclusion, preoperative physical therapy did not have a significant impact on the postoperative functional outcome of primary TKA in KOOS and ROM assessments. Patients experienced significant improvement in symptoms, pain, ADL and QOL subscales and ROM, although there was no significant improvement in the sports and recreational subscale after TKA, regardless of patients' involvement in preoperative physiotherapy. Hence, we do not recommend a more intensive course of physiotherapy regime prior to TKA surgery.

\section{ACKNOWLEDGEMENTS}

The authors would like to thank Dr Azriani Abdul Rahman from the Department of Community Medicine, Universiti Sains Malaysia, for the statistical analysis, and Mrs Anis Kausar Ghazali from the Physiotherapy Unit of HUSM. Our deepest gratitude goes to Mrs Nazmi Liana Azmi from the Clinical Research Centre, Hospital Raja Perempuan Zainab II, for her contribution to the publication of this study. An abstract of this study was presented as a poster presentation and received the Best Poster Presentation Award at Kelantan Research Day, 2013, organised by the State Clinical Research Centre.

\section{REFERENCES}

1. Fortin PR, Clarke AE, Joseph L, et al. Outcomes of total hip and knee replacement: preoperative functional status predicts outcomes at six months after surgery. Arthritis Rheum 1999; 42:1722-8.

2. Ditmyer MM, Topp MR, Pifer M. Prehabilitation in preparation for orthopaedic surgery. Orthop Nurs 2002; 21:43-51.

3. Barbay K. Research evidence for the use of preoperative exercise in patients preparing for total hip or total knee arthroplasty. Orthop Nurs 2009; 28:127-33.

4. Rodgers JA, Garvin KL, Walker CW, et al. Preoperative physical therapy in primary total knee arthroplasty. J Arthroplasty 1998; 13:414-21.

5. Weidenhielm L, Mattsson E, Broström LA, Wersäll-Robertsson E. Effect of preoperative physiotherapy in unicompartmental prosthetic knee replacement. Scand J Rehabil Med 1993; 25:33-9.

6. D'Lima DD, Colwell CW Jr, Morris BA, Hardwick ME, Kozin F. The effect of preoperative exercise on total knee replacement outcomes. Clin Orthop Relat Res 1996; 326:174-82.

7. Rooks DS, Huang J, Bierbaum BE, et al. Effect of preoperative exercise on measures of functional status in men and women undergoing total hip and knee arthroplasty. Arthritis Rheum 2006; 55:700-8

8. Ackerman IN, Bennell KL. Does pre-operative physiotherapy improve outcomes from lower limb joint replacement surgery? A systematic review. Aust J Physiother 2004; 50:25-30.

9. Roos EM, Lohmander LS. The Knee injury and Osteoarthritis Outcome 
Score (KOOS): from joint injury to osteoarthritis. Health Qual Life Outcomes 2003; 1:64.

10. Bea KC. Outcome study on glucosamine sulphate as symptom-modifying treatment in knee osteoarthritis (dissertation). Kubang Kerian, Kelantan: Kelantan Universiti Sains Malaysia, 2009.

11. Felson DT, Zhang Y, Hannan MT, et al. The incidence and natural history of knee osteoarthritis in the elderly. The Framingham Osteoarthritis Study. Arthritis Rheum 1995; 38:1500-5.

12. Peersman G, Laskin R, Davis J, Peterson M. Infection in total knee replacement: a retrospective review of 6489 total knee replacements. Clin Orthop Relat Res 2001; 392:15-23.

13. Callahan CM, Drake BG, Heck DA, Dittus RS. Patient outcomes following tricompartmental total knee replacement. A meta-analysis. JAMA 1994; 271:1349-57.

14. Ahmad Hafiz Z, Masbah O, G Ruslan. Total knee replacement: 12 years retrospective review and experience. Malays Orthop J 2011; 5:34-9.

15. Dhillon KS, Jamal A, Bhupinderjeet S. Early results of total knee replacements: "a clinical and radiological evaluation". Med J Malaysia 1993; 48:427-35.

16. Chiu KY, Ng TP, Tang WM, Yau WP. Review article: knee flexion after total knee arthroplasty. J Orthop Surg (Hong Kong) 2002; 10:194-202.

17. Kurosaka M, Yoshiya S, Mizuno K, Yamamoto T. Maximizing flexion after total knee arthroplasty: the need and the pitfalls. J Arthroplasty 2002; 17(4 Suppl 1):59-62. 